\title{
Development of an analytical model to predict oil reservoirs performance using mechanical waves propagation
}

\section{DOI : 10.36909/jer.14863}

\author{
Hesham A. Abu Zaid*, Sherif A. Akl**, Mahmoud Abu El Ela***, Ahmed El-Banbi****, and
} Mohamed H. Sayyouh**

* Belayim Petroleum Company (Petrobel), Egypt

** Cairo University, Egypt

*** Cairo University, Egypt (Corresponding Author-m.ella2003@gmail.com, m.ella@cu.edu.eg)

**** The American University in Cairo, Egypt

\begin{abstract}
The mechanical waves have been used as an unconventional enhanced oil recovery technique. It has been tested in many laboratory experiments as well as several field trials. This paper presents a robust forecasting model that can be used as an effective tool to predict the reservoir performance while applying seismic EOR technique. This model is developed by extending the wave induced fluid flow theory to account for the change in the reservoir characteristics as a result of wave application. A MATLAB program was developed based on the modified theory. The wave's intensity, pressure, and energy dissipation spatial distributions are calculated. The portion of energy converted into thermal energy in the reservoir is assessed. The changes in reservoir properties due to temperature and pressure changes are considered. The incremental oil recovery and reduction in water production as a result of wave application are then calculated. The developed model was validated against actual performance of Liaohe oil field. The model results show that the wave application increases oil production from 33 to 47 ton/day and decreases water-oil ratio from 68 to $48 \%$, which is close to the field measurements. A parametric analysis is performed to identify the important parameters that affect reservoir performance under seismic EOR. In addition, the study determines the optimum ranges of reservoir properties where this technique is most beneficial.
\end{abstract}

Keywords: Downhole Seismic Tools; In-Situ Seismic; Mechanical Waves; Seismic EOR 


\section{INTRODUCTION}

Since 1954, many theories have been developed to understand the full image of elastic wave propagation through saturated elastic porous media. The first formulation of elastic wave propagation in porous media was postulated by Biot (1965a, 1965b). He developed a theory for the propagation of stress waves in a porous elastic solid media saturated by a viscous fluid.

Later, and starting from the early 1980's until now, the interest in applying elastic wave stimulation was focused mainly on acoustic and to a lower degree on surface vibrators. In the late 1990's, a third type of elastic wave stimulation (in-situ seismic stimulation) was developed. The mechanism is to create a highenergy elastic wave identical to earthquakes but at reservoir depth (in-situ) and lower magnitudes so as not to destroy the reservoir (Kostrov \& Wooden, 2008). Extensive field studies using downhole seismic stimulation have proven to increase oil production and oil recovery.

The theories developed after Biot addressed the limitations of this theory's assumptions. These theories extended Biot's theory to cover all frequency ranges and two-phase flow type (Brutsaret, 1964), doubleporosity effect (Ba et. al., 2011), squirt flow model (Mavko \& Jizba, 1991; Chapman et. al., 2002), saturation fluid effect (Calvert, 2005), patchy saturation effect (Mavko \& Mukerji, 1998), and the use of spatially varying coefficients (Müller \& Gurevich, 2005a; Müller \& Gurevich, 2005b)

These theories rely on some restricting assumptions limiting their application. The common assumptions: (1) homogeneous reservoir, (2) one-dimensional wave flow propagation, (3) single-phase fluid, and (4) no thermo-elastic effects. In addition, the main problem of these theories is that it focuses on the wave properties and ignores the wave's subsequent effects on reservoir properties. They also ignore the changes in reservoir performance resulting from wave application.

On the other hand, the literature includes several recent laboratory studies which have been performed to study the effect of acoustic waves on the hydrocarbon properties and flow behavior in porous media (Hamidi et. al., 2014; Hamidi et. al., 2015; Louhenapessy \& Ariadji, 2020; Khasi et. al., 2021). 
In this paper, a model is developed by combining multidiscipline scientific branches to predict the reservoir performance while applying seismic EOR technique. Wave motion and propagation in rocks and its energy distribution and dissipation or attenuation is studied. In addition, the wave induced fluid flow theory is modified to consider the changes in reservoir characteristics caused by exposing the reservoir to mechanical waves. The model calculates the spatial distribution of the wave's intensity, pressure, and energy dissipation. Moreover, it assumes that the portion of energy dissipated to the reservoir is converted into thermal energy. The developed model can calculates the change in reservoir properties due to temperature and pressure changes. The incremental oil recovery and reduction in water production as a result of wave application is then calculated. A simple MATLAB program is developed to implement this analytical model.

\section{MECHANICAL WAVES STIMULATION MECHANISM}

There are several possible mechanisms that attempt to explain oil extraction from porous media under the effect of high energy elastic waves. These waves cause mechanical disturbance in the reservoir fluids while they move. The mechanical disturbance continues to consume the wave power until relaxation occurs. High energy elastic waves introduce mechanical vibrations, which strongly influence interfacial and viscous forces by enhancing momentum and heat transfer across the phase interfaces. These effects are believed to be responsible for the observed improvement in percolation of oil within porous media (Hamida \& Babadagli, 2005; Hamida \& Babadagli, 2007). These effects strongly depend on the elastic wave's frequency and intensity. Intensity defines the wave strength and quantifies both the wave mechanical and thermal impacts. Frequency plays an important role in wave dispersion, attenuation, and penetration profile into the formation.

\section{MODEL FORMULATION}

A reliable theory that applied the method of statistical smoothing to Biot's poroelasticity equations in a random, heterogeneous, porous medium has been developed and named the wave induced fluid flow theory (Müller \& Gurevich, 2005a; Müller \& Gurevich, 2005b). The fast and slow P-wave velocities, inverse quality factor, attenuation, and propagation schemes are derived assuming that the reservoir consists of a single homogeneous background rock type with small discrete patches of other rock types. The statistical smoothing method is used to account for these discrete patches, the spatial distribution of these rocks is modeled by a 
unified spatial correlation function $\mathrm{B}(\mathrm{r})$. This function is the variance of the random relative fluctuations in rock properties. All poroelastic parameters assumed to have the same unified spatial function and correlation length. The effective $\mathrm{P}$-wave number $\bar{K}_{P}$ is derived to be:

$$
\bar{K}_{P}=K_{P}\left(1+\Delta_{2}+\Delta_{1} K_{P S}^{2} \int_{0}^{\infty} r B(r) \exp \left(\text { ir } K_{P S}\right) d r\right) \quad \text { Eq. } 1 .
$$

Where the dimensionless coefficients $\Delta_{1}$ and $\Delta_{2}$ are given by:

$$
\begin{array}{cc}
\Delta_{1}=\frac{\alpha^{2} M}{2 P_{d}}\left(\sigma_{H H}^{2}-2 \sigma_{H C}^{2}+\sigma_{C C}^{2}+\frac{32}{15} \frac{G^{2}}{H^{2}} \sigma_{G G}^{2}-\frac{8}{3} \frac{G}{H} \sigma_{H G}^{2}+\frac{8}{3} \frac{G}{H} \sigma_{G C}^{2}\right) & \text { Eq. } 2 . \\
\Delta_{2}=\Delta_{1}+\frac{1}{2} \sigma_{H H}^{2}-\frac{4}{3} \frac{G}{H} \sigma_{H G}^{2}+\left(\frac{4 G}{H}+1\right) \frac{4}{15} \frac{G}{H} \sigma_{G G}^{2} & \text { Eq. } 3 .
\end{array}
$$

Where

$$
\begin{array}{cc}
B(0)=1 & B(\infty)=0 \\
K_{P s}=\sqrt{i \omega \mu /\left(k_{0} N\right)} & H=P_{d}+\alpha^{2} M \\
M=\left[(\alpha-\phi) / K_{0}+\phi / K_{f}\right]^{-1} & \alpha=1-K_{d} / K_{0} \\
N=M P_{d} / H & C=\alpha M
\end{array}
$$

Expressions for velocity dispersion $V(\omega)$ and attenuation or inverse quality factor $Q^{-1}$ are obtained from the real and imaginary parts of the effective wavenumber:

$$
\begin{gathered}
V(\omega)=\frac{\omega}{\operatorname{Re}\left\{\bar{K}_{P}\right\}}=V_{0}\left(1-\Delta_{2}+2 \Delta_{1} K_{P s r}^{2} \int_{0}^{\infty} r B(r) \exp \left(-r K_{P s r}\right) \sin \left(r K_{P s r}\right) d r\right) \\
Q^{-1}(\omega)=\frac{2 \operatorname{Im}\left\{\bar{K}_{P}\right\}}{\operatorname{Re}\left\{\bar{K}_{P}\right\}}=4 \Delta_{1} K_{P s r}^{2} \int_{0}^{\infty} r B(r) \exp \left(-r K_{P s r}\right) \cos \left(r K_{P s r}\right) d r
\end{gathered}
$$

Where

$$
V_{0}=\sqrt{H / \rho}=\text { constant } \quad K_{P s r}=\sqrt{\omega \mu /\left(2 k_{0} N\right)}
$$

The limitation of this model is that it homogenized the whole reservoir into a single rock type by using the statistical smoothing approach. In addition, all the previous wave-propagation theories are studied for earthquakes and petroleum exploration modeling purposes. They did not consider the wave effects on the reservoir fluids. They only focused on the wave propagation, intensity, and strength. The purpose of this study is to modify Müller and Gurevich's model and extend it to account for the effect of the wave on reservoir 
rock and fluid properties. In addition, it takes into consideration the wave energy effects on enhancing petroleum reservoirs performance.

The following modifications are applied to the original Müller and Gurevich's model in order to simulate and forecast the reservoir performance under the application of the seismic wave EOR technique:

1. Wave Attenuation Calculations in $d B$ : The quality factor, $\mathrm{Q}$, is a measure of the material resistance to wave propagation. The quality factor describes how strong the wave is. The attenuation coefficient or the attenuation rate $(\overline{\boldsymbol{\alpha}})$ is a function of the quality factor and the wavelength (Wang, 2008). The wave attenuation at any location $r$ away from the wave-source center is calculated as shown in Eq. 6 .

$$
\text { Attenuation }=\bar{\alpha} * r=\frac{Q^{-1} *(27.3)}{\lambda} * r=\frac{Q^{-1} *(27.3)}{(2 * \pi * V) / \omega} * r \quad \text { (dB) } \quad \text { Eq. } 6 \text {. }
$$

2. Wave intensity and Pressure Distribution Calculations: Seismic waves transport energy from one point to another through a transporting medium that acts as the resistance medium to the wave propagation and successively consumes its energy until the wave vanishes. Seismic quantities such as intensity or pressure are usually quantified on a logarithmic scale and measured by decibels $(\mathrm{dB})$. The wave intensity and pressure at any point with reference to that at $1 \mathrm{~m}$ from the wave source as a function of wave attenuation could be expressed by Eq. 7, and Eq. 8 respectively (Lurton, 2010).

$$
\begin{array}{ll}
\text { Intensity }=\text { Intensity }_{1 m} * 10^{-\left(\frac{\text { Attenuation }}{10(d B)}\right)} & \text { Eq. } 7 . \\
\text { pressure }=\text { pressure }_{1 m} * 10^{-\left(\frac{\text { Attenuation }}{20(d B)}\right)} & \text { Eq. } 8 .
\end{array}
$$

3. Wellbore Losses Calculations: The losses in the wellbore arise from the reflected part of the wave on the surface of the reservoir rock. When a wave travels from one medium (wellbore fluid) to another medium different in density (reservoir rock), the wave is divided into two parts (reflected part and transmitted part) obeying the well-known Snell's law. The transmission coefficient could be calculated using the formula expressed in Eq. 9.

$$
\tau=\frac{2 z_{1}}{z_{1}+z_{2}} \quad \text { where } \quad z=\rho v \quad \text { Eq. } 9 .
$$


4. Temperature Change Calculations: Figure 1 shows a wave moving between two points 1 and 2 in the reservoir. The wave's energy decreases from original energy $\boldsymbol{e}_{\mathbf{1}}$ to the final energy $\boldsymbol{e}_{2}$. Similarly, the wave's power decreases from original power $\boldsymbol{P}_{\mathbf{1}}$ to the final power $\boldsymbol{P}_{\mathbf{2}}$ starting at the original time $\boldsymbol{t}_{\mathbf{1}}$ and ending at the final time $\boldsymbol{t}_{\mathbf{2}}$. The change in wave's energy $\Delta \boldsymbol{e}$ is calculated and converted into heat energy which is responsible for raising the reservoir's liquids temperature as expressed in Eq. 10.

$$
\Delta e=\Delta P * \Delta t \quad \text { Eq. } 10 \text {. }
$$

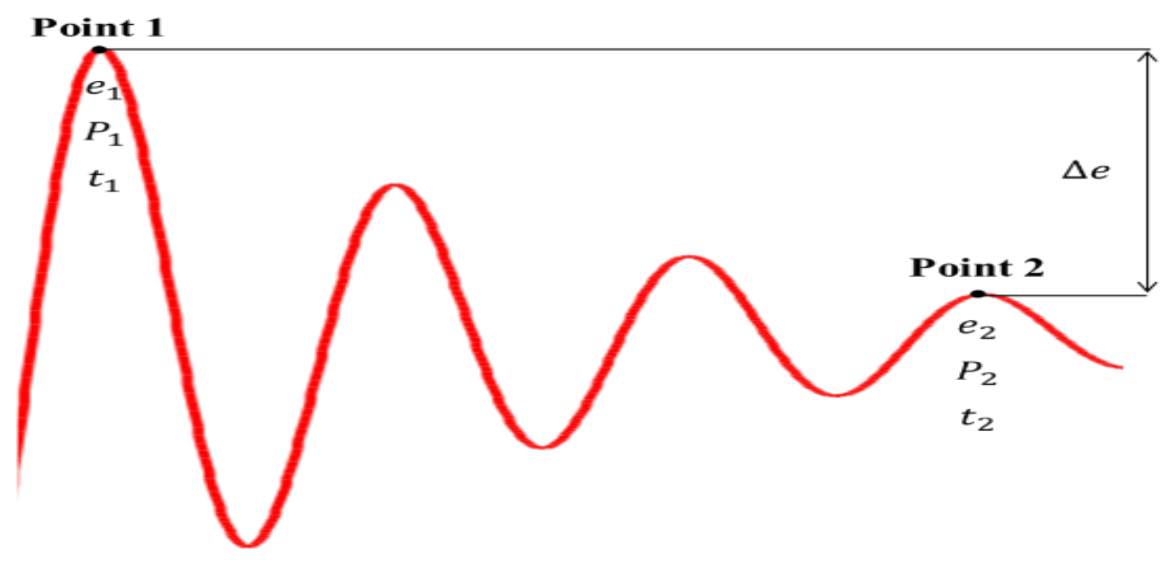

Figure 1. Wave power/energy loss while moving from Point 1 to Point 2.

Energy added to the reservoir is converted to heat energy following the principle of energy conservation. Heat energy raises the reservoir fluid temperature following the well-known heat transfer equation (Eq. 11).

$$
q_{t h}=m c \Delta T
$$

Applying the energy conservation principle (the energy lost from the wave is equal to the energy added to the reservoir), Eq. 12 is composed.

$$
\Delta e=\Delta P * \Delta t=q_{t h}=m c \Delta T
$$

Then, convection heat transfer from reservoir fluid to reservoir rock occurs following the convective heat transfer given by Eq. 13 .

$$
q_{t h}=h_{c} A \Delta T
$$

Mass and heat balance need to be checked at every grid block for every time step. At each time step, a certain mass of fluid exits, and the rest of the grid block fluid mass remains, while another mass is added. 
Mass balance is applied as in Eq. 14. Heat balance (thermal equilibrium) is applied taking into consideration the mass balance for the grid block.

$$
\text { Final fluid mass }=\text { added fluid mass }+ \text { remaining fluid mass } \quad \text { Eq. } 14 \text {. }
$$

Thermal losses occurred at reservoir boundaries through conduction to overburden and underlying rock layers. The thermal energy flow rate is calculated using Fourier's law which is expressed in Eq. 15.

$$
q_{t h}=-\Lambda A \frac{\Delta T}{d} \quad \text { Eq. } 15 \text {. }
$$

The thermal flow rate calculated in Eq. 15 is deducted from the reservoir thermal energy and added to the overburden and underlying rock layers' thermal energy. The final reservoir rock and fluid temperature is calculated based on the deducted thermal energy.

5. Flow Rate Calculations: Based on the data provided by Arps (1956), a correlation that relates the residual oil saturation with the oil viscosity both under reservoir conditions is established as presented in Eq. 16 with R-squared value of 0.9983.

$$
S_{\text {or }}=3.9201 * \operatorname{Ln}\left(\mu_{o}\right)+34.487 \quad \text { Eq. } 16 \text {. }
$$

Decreasing oil viscosity causes some of the residual oil saturation to become mobile, which increases oil recovery. In addition, it increases the mobility of oil relative to that of water, which accelerates the recovery and decreases the water cut. Wave propagation through the reservoir changes two parameters in Darcy's equation (Eq. 17). It decreases capillary pressure by acting against it with a value of the wave pressure $p_{\text {wave }}$. In addition, it decreases the oil viscosity $\mu_{o}$. These two effects increase the flow rate and the reservoir recovery. The effect of wave pressure on capillary pressure was modeled by assuming that the wave pressure $p_{\text {wave }}$ must exceed the value of capillary pressure to be able to free the oil droplet from the holding capillary forces. The temperature effect was assumed in the model by updating oil viscosity value as function of temperature.

$$
q=\frac{0.00708 * k h *\left(\bar{p}-p_{w f}-P_{c}\right)}{\mu * B *\left[\ln \left(\frac{r_{e}}{r_{w}}\right)-0.75+s\right]}
$$


The existence of free gas at the reservoir will weaken the application of this method. The free gas will act as an air bag that absorbs the wave impact. Therefore, seismic EOR is better applied to an oil reservoir above the bubble point pressure.

\section{PROGRAMMING AND PARAMETRIC STUDY}

The developed model was programmed using MATLAB programming language. The code flowchart is presented in Figure 2. The code calculates the average rock and fluid properties of the grid block such as the average porosity, permeability, fluid saturations, fluid density, rock density, fluid viscosity, and bulk modulus. The code calculates the wave velocity, the wave quality factor, the wave attenuation in $\mathrm{dB}$, and the wave intensity and pressure in every grid block.

To calculate the effect of the waves on production, the code calculates the energy added to the grid block at each time step, the consequent change in the grid block temperature, and the change in the grid block fluids density and viscosity. The code applies Darcy's equation and the material balance equation for fluid flow and mass conservation. All these calculations can run with and without applying the wave effects to investigate the effect of the wave application.

At the end of the run, a detailed report of the run is printed as well as an output data file. A group of figures is presented to illustrate the effect of applying the wave on the performance of the reservoir. These figures compare between the production with and without wave application.

A parametric study is conducted to investigate the effects of the wave parameters, reservoir rock and fluid properties, and wellbore fluid density. In addition, the comparison between reservoir performance with and without wave application is useful in identifying the most influential parameters on the application of seismic EOR technique. Moreover, this comparison could specify the optimum properties ranges for the application of the technique.

The parametric study shows that the most influential parameters are the wave frequency, original intensity, the wellbore fluid density, and the distance between the producing well and the wave source. In addition, reservoir initial temperature, permeability, and oil density are critical parameters. On the other hand, the average reservoir porosity is not a critical parameter. The optimum range of these critical parameters in 
order to achieve the best reservoir performance under the application of high energy elastic waves as an EOR agent is listed in Table 1.

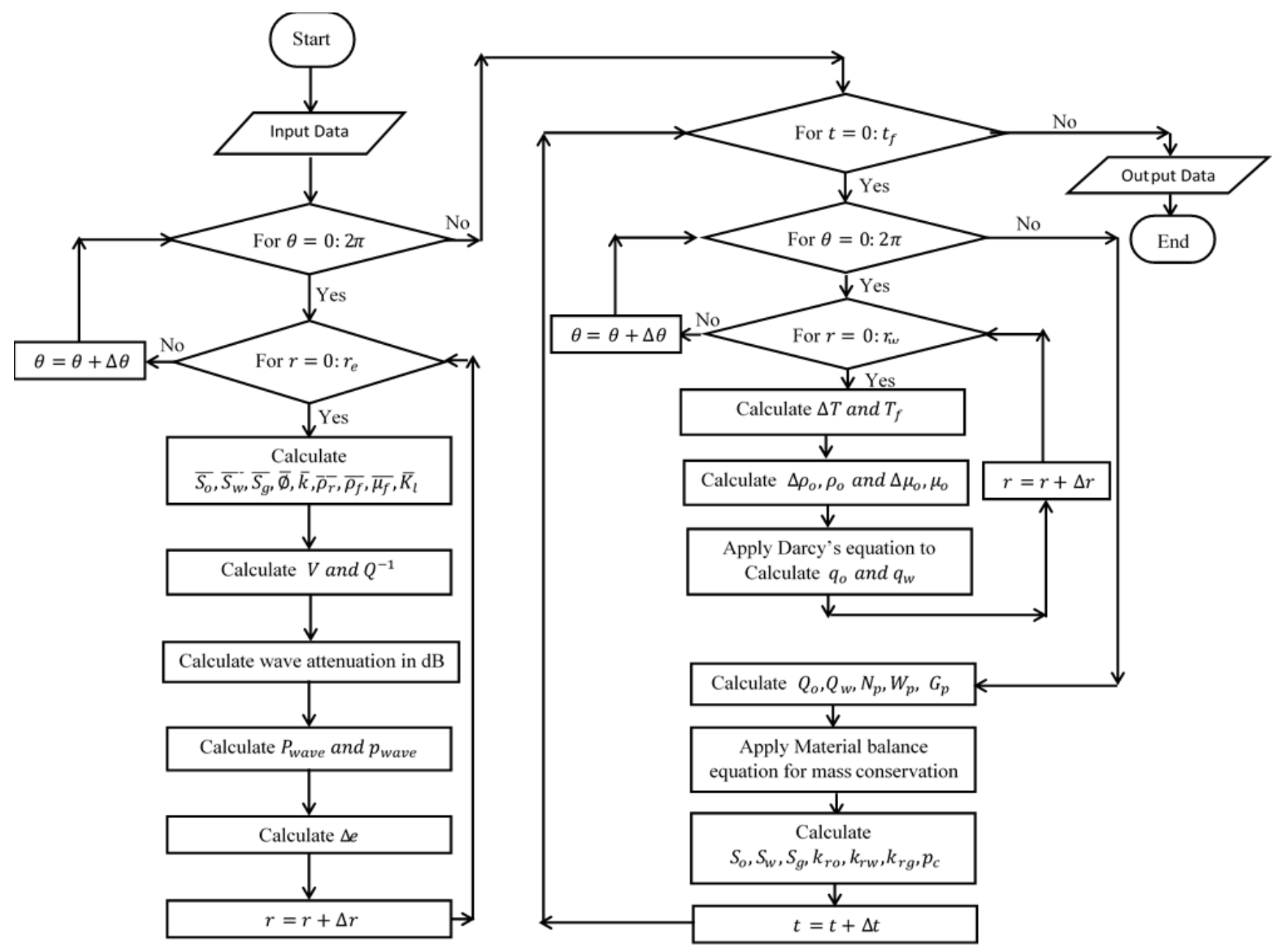

Figure 2. The model programming code flowchart

Table 1. The optimum range of the critical controlling parameters

\begin{tabular}{|c|c|}
\hline Controlling Parameter & Optimum Range \\
\hline Wave Frequency & $100: 1000 \mathrm{~Hz}$ \\
\hline Wave intensity & $>100 \mathrm{watt} / \mathrm{m}^{2}$ \\
\hline Distance from Source & $<1000 \mathrm{~m}$ \\
\hline Reservoir Permeability & $>10 \mathrm{md}$ \\
\hline Reservoir Oil Density & $800: 1000 \mathrm{Kg} / \mathrm{m}^{3}$ \\
\hline Initial Reservoir Temperature & $<50^{\circ} \mathrm{C}$ \\
\hline Wellbore Fluid Type & High density liquids \\
\hline Reservoir Porosity & Not critical \\
\hline
\end{tabular}




\section{MODEL VALIDATION}

Downhole seismic EOR technique was tested in Liaohe oilfield for 20 days (Zhu et. al., 2005). The developed model was used to simulate the performance of Liaohe oilfield test. The wave source and the actual reservoir parameters are used as input data for the program. The results of the program are compared with the actual test results.

It should be highlighted that the Liaohe oilfield was in late cyclic steam stimulated recovery period before applying the downhole vibration tool. The wave source was Downhole Harmonic Vibration Oildisplacement System (DHVOS) tool. The wave source performs 88 shocks per minute with each shock having 5-ton strength. The accumulated vibration test was continued for 20 days. A total of 23 oil wells were effectively influenced by the artificial seismic waves within the 300:400-meter radius around the seismic source well. The oil production increased from 36 tons/day before using the tool to 47 tons/day while applying the wave. The cumulative water cut in the stimulated area declined from $68 \%$ before using the tool to $56 \%$ while using the tool.

The developed program was run using the properties of Liaohe reservoir (Table 2). The results of the model are presented in Figures 3 and 4.

Table 2. Summary of the input data of Liaohe oilfield case

\begin{tabular}{|c|c|c|}
\hline \multicolumn{2}{|c|}{ Input Data Provided in the Original Publication by Zhu et al. (2005) } \\
\hline Parameter & Value & Dimensions \\
\hline Pay Thickness & 67.7 & $\mathrm{~m}$ \\
\hline Porosity & 24 & $\mathrm{md}$ \\
\hline Permeability & 2420 & $\mathrm{mPa} . \mathrm{s}$. \\
\hline Crude oil viscosity & 500 & $\mathrm{gm} / \mathrm{cm}^{3}$ \\
\hline Crude oil density & 0.946 & $\mathrm{Watt} / \mathrm{m}^{2}$ \\
\hline Wave intensity & 1483 & $\mathrm{psi}$ \\
\hline Wave source pressure & 147 & $\mathrm{day}$ \\
\hline Application period & 20 & $\mathrm{well}$ \\
\hline Affected wells & 23 & $\mathrm{~m}$ \\
\hline Wells distribution radius & $300: 400$ & $\mathrm{psi}$ \\
\hline & Assumed Data & $\mathrm{psi}$ \\
\hline Average reservoir pressure & 914 & ${ }^{\circ} \mathrm{C}$ \\
\hline Bottomhole flowing pressure & 880 & \\
\hline Reservoir temperature & 23 & \\
\hline
\end{tabular}




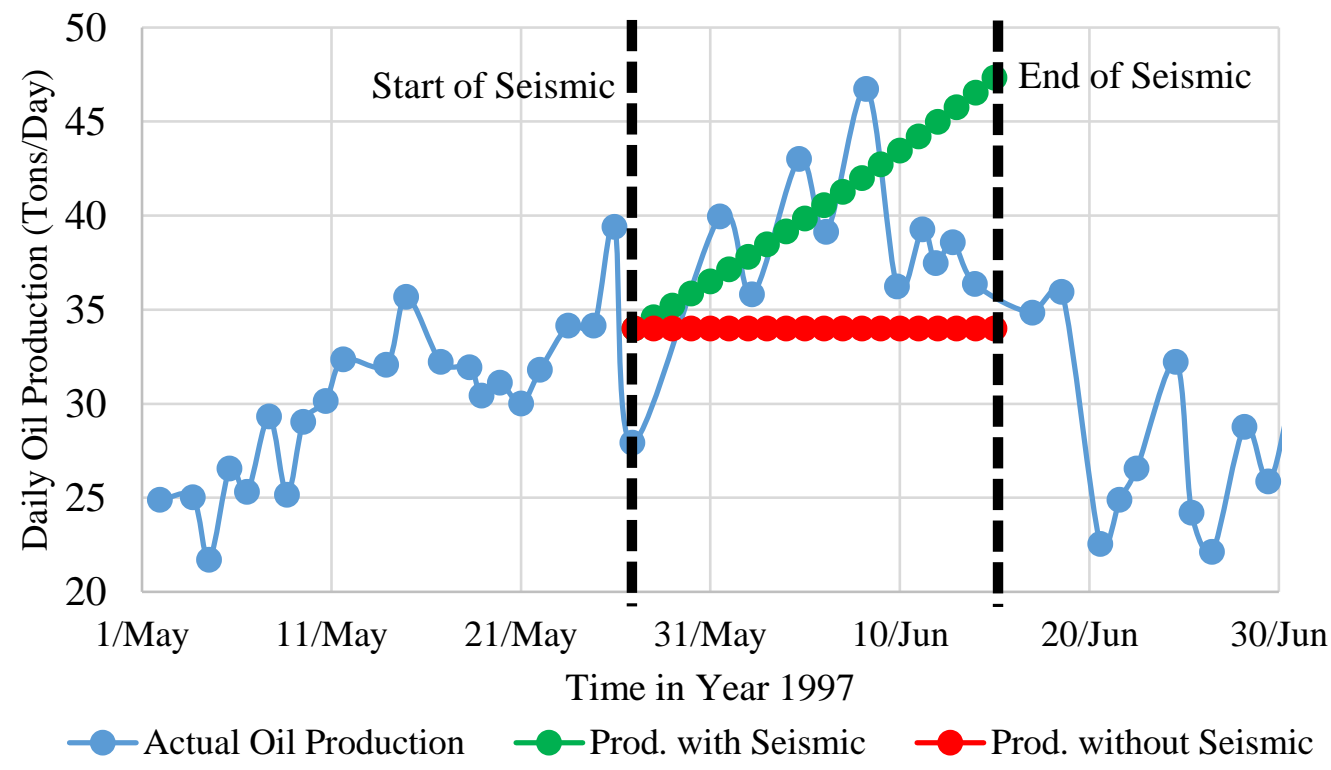

Figure 3. The daily oil production with and without the wave application using the developed model Vs Liaohe oilfield data in the range 300-400m (Total 23 wells)

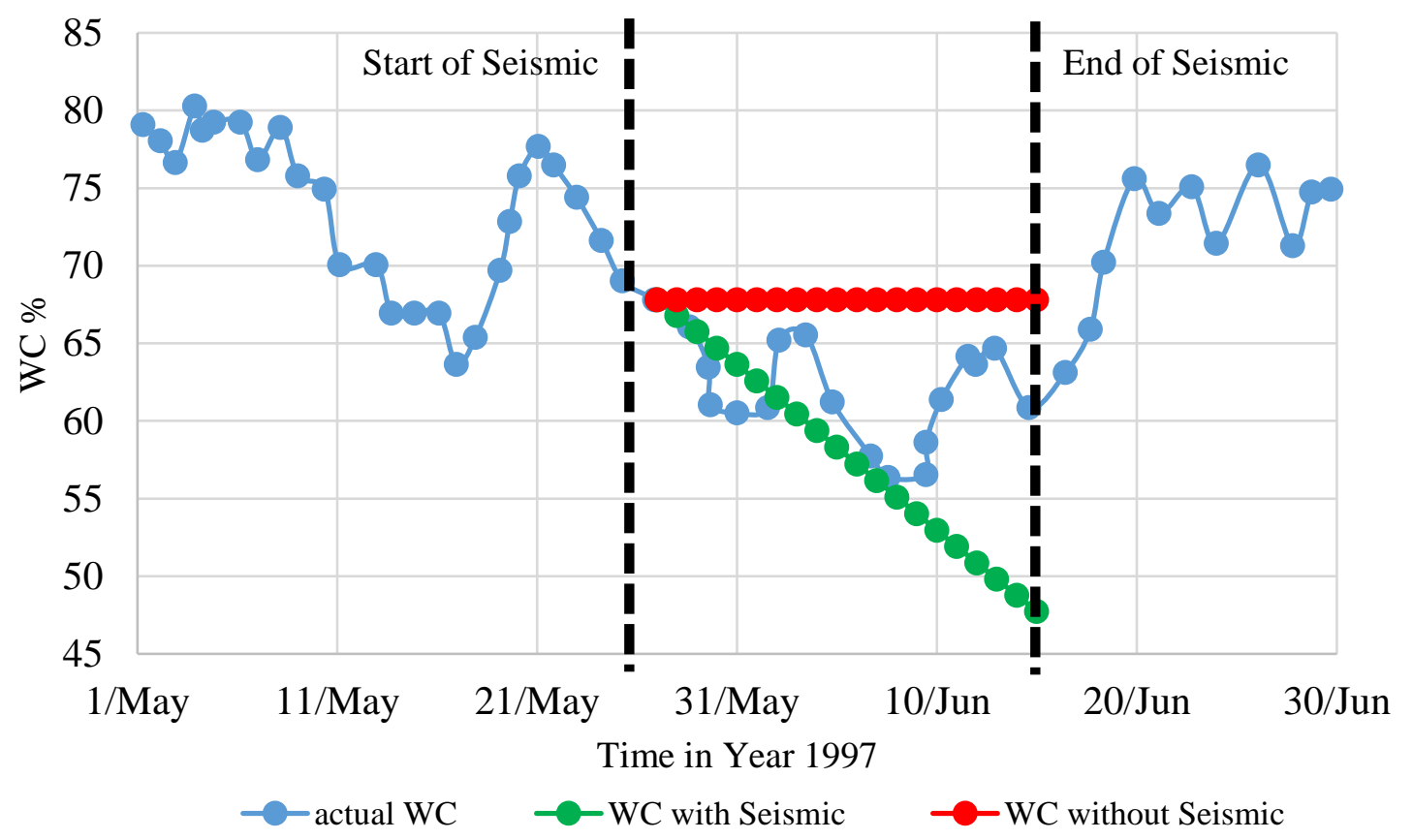

Figure 4. The water cut with and without the wave application using the developed model Vs Liaohe oilfield data in the range 300-400m (Total 23 wells)

The model application in the range of 300:400-meter radius around the wave source results in an increase in oil production rate from 33 to 47 ton/day and a decrease in the water-oil ratio from 68 to $48 \%$. This increase 
in oil production and decrease in the water-oil ratio are relatively close to the actual field observations (actual field observation shows increase in oil production from 36 to 47 tons/day and decrease in water-oil ratio from 68 to 56\%). This reasonable match verifies the reliability of the developed model.

The results of running the new developed program using Liaohe oilfield test properties shows that the wave intensity and wave pressure are exponentially decreasing at different orders with distance from the source by the act of the increasing wave attenuation. In this case, the reservoir absorbs most of the wave power and pressure around $1000 \mathrm{~m}$. The zone near the wave source is exposed to a very high wave pressure and absorbs most of the wave energy. Hence, zones near the wave source are expected to have the greatest improvement in the reservoir performance. These results agree with the empirical observation that downhole seismic application is only effective within a particular distance of the tool (Kostrov \& Wooden, 2008).

\section{CONCLUSIONS}

1. A new model has been developed to simulate reservoir performance under the application of high energy mechanical waves.

2. The developed model was validated against actual performance of Liaohe oilfield test case.

3. The study showed that the mechanical waves have minor effect on wells at distances more than 1000 $\mathrm{m}$ from the source.

4. The wellbore loss consumes about $70 \%$ of the original wave strength and this percentage depends on wellbore fluid type. Denser wellbore fluids allow more of the wave to penetrate the reservoir.

5. The wave frequency dictates both penetration radius and profile. Frequencies higher than $1000 \mathrm{~Hz}$ limit the wave penetration radius to a few meters into the reservoir. 


\section{NOMENCLATURE}

\begin{tabular}{|c|c|c|c|c|c|}
\hline Symbol & Definition & Unit & Symbol & Definition & Unit \\
\hline$A$ & The contact surface area & $\mathrm{m}^{2}$ & $r$ & The distance from the wave source & $\mathrm{m}$ \\
\hline$B$ & Fluid formation volume factor & bbl/STB & $r_{e}$ & Well drainage radius & $\mathrm{ft}$ \\
\hline$B(r)$ & The normalized correlation function & Dimensionless & $r_{w}$ & Wellbore radius & $\mathrm{ft}$ \\
\hline$c$ & The fluid heat capacity & $\mathrm{J} / \mathrm{Kg} .{ }^{\circ} \mathrm{C}$ & $S$ & Skin factor & Dimensionless \\
\hline $\mathrm{d}$ & The rock layer thickness & $\mathrm{m}$ & $S_{\text {or }}$ & Residual oil saturation & $\%$, Dimensionless \\
\hline$h$ & Formation thickness & $\mathrm{ft}$ & $T$ & The crude oil temperature & ${ }^{\circ} \mathrm{C}$ \\
\hline$h_{c}$ & The convective heat transfer coefficient & $\mathrm{J} / \mathrm{m}^{2} .{ }^{\circ} \mathrm{C}$ & $\mathrm{V}$ & The wave velocity & $\mathrm{m} / \mathrm{s}$ \\
\hline$k$ & Absolute permeability & md & $V_{0}$ & $\begin{array}{c}\text { The constant background } \mathrm{P} \text {-wave } \\
\text { velocity }\end{array}$ & $\mathrm{m} / \mathrm{s}$ \\
\hline$K_{d} K_{f}$ & The dry and fluid bulk modulus respectively & $\mathrm{Pa}$ & $\mathrm{Z}$ & The impedance & $\mathrm{Kg} / \mathrm{m}^{2} . \mathrm{s}$ \\
\hline$K_{P s r}$ & The wave number of Biot's slow wave & Dimensionless & Greek & Definition & Unit \\
\hline$k_{0}$ & The background permeability & $\mathrm{md}$ & $\alpha$ & The Biot-Willis coefficient & Dimensionless \\
\hline$m$ & The fluid mass & $\mathrm{Kg}$ & $\bar{\alpha}$ & The attenuation coefficient & $\mathrm{dB} / \mathrm{m}$ \\
\hline$p$ & Volumetric average reservoir pressure & psi & $\lambda$ & The wavelength & $\mathrm{m}$ \\
\hline$P_{1 m}$ & $\begin{array}{l}\text { The wave power at a distance one meter } \\
\text { from the source }\end{array}$ & watts & $\Delta_{1}, \Delta_{2}$ & Special coefficients & Dimensionless \\
\hline$p_{1 m}$ & $\begin{array}{l}\text { The wave pressure at a distance one meter } \\
\text { from the source }\end{array}$ & psi & $\mu$ & Fluid viscosity & $\mathrm{cp}$ \\
\hline$p_{c}$ & Capillary pressure & psi & $\mu_{o}$ & Oil viscosity & cp \\
\hline$P_{d}$ & The dry background P-wave modulus & Dimensionless & $\Lambda$ & The rock thermal conductivity & $\mathrm{W} / \mathrm{m} .{ }^{\circ} \mathrm{C}$ \\
\hline$p_{e}$ & Average reservoir pressure & psi & $\phi$ & The background porosity & $\%$, Dimensionless \\
\hline$p_{\text {wave }}$ & Wave pressure & psi & $\tau$ & The transmission coefficient & $\%$, Dimensionless \\
\hline$p_{w f}$ & Bottom hole flowing pressure & psi & $\omega$ & The wave angular frequency & $\mathrm{Hz}$ \\
\hline$Q^{-1}$ & Wave Quality Factor & Dimensionless & $\rho$ & The saturated bulk density & $\mathrm{Kg} / \mathrm{m}^{3}$ \\
\hline$q$ & Flow rate & bbl/day & & & \\
\hline
\end{tabular}

\section{REFERENCES}

Arps, J.J. 1956. Estimation of primary oil reserves. Journal of Petroleum Technology 207:182-191

Ba, J., Carcione, J.M. \& Nie, J.X. 2011. Biot-Rayleigh theory of wave propagation in double-porosity media. Journal of Geophysical Research: Solid Earth 116 (B06202):1-12. https://doi.org/10.1029/2010JB008185

Biot, M. A. 1956a. Theory of propagation of elastic waves in fluid-saturated porous solid. I. Low-frequency range. The Journal of the Acoustical Society of America 28(2):168-178. https://doi.org/10.1121/1.1908239 
Biot, M. A. 1956b. Theory of propagation of elastic waves in a fluid-saturated porous solid. II. Higher frequency range. The Journal of the Acoustical Society of America, 28(2):179-191. https://doi.org/10.1121/1.1908241

Brutsaret, W. 1964. The propagation of elastic waves in unconsolidated unsaturated granular mediums. Journal of Geophysical Research 69(2): 243-257. https://doi.org/10.1029/JZ069i002p00243

Calvert, R. 2005. Insights and Methods for 4D Reservoir Monitoring and Characterization. Society of Exploration Geophysicists and European Association of Geoscientists and Engineers. https://doi.org/10.1190/1.9781560801696

Chapman, M., Zatsepin, S.V. \& Crampin, S. 2002. Derivation of a microstructural poroelastic model. Geophysical Journal International 151(2):427-451.https://doi.org/10.1046/j.1365-246X.2002.01769.x

Hamida, T. \& Babadagli, T. 2005. Effects of ultrasonic waves on immiscible and miscible displacement in porous media. SPE-95327-MS, SPE Annual Technical Conference and Exhibition. Society of Petroleum Engineers. 9-12 October, Dallas, Texas, USA. https://doi.org/10.2118/95327-MS

Hamida, T. \& Babadagli, T. 2007. Immiscible displacement of oil by water in consolidated porous media due to capillary imbibition under ultrasonic waves. The Journal of the Acoustical Society of America 122(3): 1539-1555.

Hamidi, H., Mohammadian, E., Junin, R., Rafati, R., Manan, M., Azdarpour, A. \& Junid, M. 2014. A technique for evaluating the oil/heavy-oil viscosity changes under ultrasound in a simulated porous medium. Ultrasonics, 54(2), pp.655-662. https://doi.org/10.1016/j.ultras.2013.09.006

Hamidi, H., Mohammadian, E., Asadullah, M., Azdarpour, A. \& Rafati, R. 2015. Effect of ultrasound radiation duration on emulsification and demulsification of paraffin oil and surfactant solution/brine using Hele-shaw models. Ultrasonics Sonochemistry, Volume 26 (September 2015), pp.428-436. https://doi.org/10.1016/j.ultsonch.2015.01.009

Khasi, S., Fayazi, A. \& Kantzas, A. 2021. A pore-scale model for dispersion and mass transfer during acoustically assisted miscible displacements in porous media. Industrial \& Engineering Chemistry Research, 60(4), pp.1884-1900. https://doi.org/10.1021/acs.iecr.0c05141 
Kostrov, S.A. \& Wooden, W.O. 2008. Possible mechanisms and cases for enhancement of oil recovery and production using seismic stimulation. SPE Symposium on Improved Oil Recovery. Society of Petroleum Engineers. SPE-114025-MS, 22-23 April, Tulsa, Oklahoma, USA. https://doi.org/10.2118/114025-MS

Louhenapessy, S.C. \& Ariadji, T. 2020. The effect of type waves on vibroseismic implementation of changes properties of rock, oil viscosity, oil compound composition, and enhanced oil recovery. Petroleum Research, 5(4), pp.304-314. https://doi.org/10.1016/j.ptlrs.2020.05.001

Lurton, X., 2010. An Introduction to Underwater Acoustics: Principles and Applications (Chapter 2: Underwater Acoustic Wave Propagation), $2^{\text {nd }}$ edition, Springer Berlin Heidelberg, pp.13-23. https://www.springer.com/gp/book/9783540784807

Mavko, G. \& Jizba, D. 1991. Estimating grain-scale fluid effects on velocity dispersion in rocks. Geophysics 56(12): 1940-1949. http://dx.doi.org/10.1190/1.1443005

Mavko, G. \& Mukerji, T. 1998. Bounds on low-frequency seismic velocities in partially saturated rocks. Geophysics 63(3): 918-924. https://doi.org/10.1190/1.1444402

Müller, T.M. \& Gurevich, B. 2005a. A first-order statistical smoothing approximation for the coherent wave field in random porous media. The Journal of the Acoustical Society of America 117(4): 1796-1805. https://doi.org/10.1121/1.1871754

Müller, T.M. \& Gurevich, B. 2005b. Wave-induced fluid flow in random porous media: Attenuation and dispersion of elastic waves. The Journal of the Acoustical Society of America 117(5): 2732-2741. https://doi.org/10.1121/1.1894792

Wang, Y. 2008. Seismic Inverse Q Filtering (Chapter 1: Introduction to Inverse Q Filtering). John Wiley \& Sons, pp.2-4. https://www.wiley.com/en-al/Seismic+Inverse+Q+Filtering-p-9781405185400

Zhu, T., Huang, X. \& Vajjha, P.K. 2005. Downhole harmonic vibration oil-displacement system: a new IOR tool. SPE Western Regional Meeting. SPE-94001-MS, Irvine, California, 30 March - 1 April. https://doi.org/10.2118/94001-MS 